\title{
Fluorescence nanoscopy by polarization modulation and polarization angle narrowing
}

\author{
Nour Hafi ${ }^{1,8}$, Matthias Grunwald ${ }^{2,8}$, Laura S van den Heuvel ${ }^{1,8}$, Timo Aspelmeier ${ }^{3-5,8}$, Jian-Hua Chen ${ }^{2}$, \\ Marta Zagrebelsky ${ }^{6}$, Ole M Schütte ${ }^{7}$, Claudia Steinem ${ }^{7}$, Martin Korte ${ }^{6}$, Axel Munk ${ }^{3-5}$ \& Peter J Walla ${ }^{1,2}$
}

\begin{abstract}
When excited with rotating linear polarized light, differently oriented fluorescent dyes emit periodic signals peaking at different times. We show that measurement of the average orientation of fluorescent dyes attached to rigid sample structures mapped to regularly defined $(50 \mathrm{~nm})^{2}$ image nanoareas can provide subdiffraction resolution (super resolution by polarization demodulation, SPoD). Because the polarization angle range for effective excitation of an oriented molecule is rather broad and unspecific, we narrowed this range by simultaneous irradiation with a second, de-excitation, beam possessing a polarization perpendicular to the excitation beam (excitation polarization angle narrowing, ExPAN). This shortened the periodic emission flashes, allowing better discrimination between molecules or nanoareas. Our method requires neither the generation of nanometric interference structures nor the use of switchable or blinking fluorescent probes. We applied the method to standard wide-field microscopy with camera detection and to two-photon scanning microscopy, imaging the fine structural details of neuronal spines.
\end{abstract}

In recent years the development of super-resolution techniques has had a profound impact on biology and other fields in which subdiffraction-limited resolution of fluorescently labeled samples is desired ${ }^{1-13}$. Prominent examples are stimulated emission depletion (STED) microscopy ${ }^{1,4}$, photoactivated localization microscopy (PALM) ${ }^{3,5,6}$ and stochastic optical reconstruction microscopy (STORM) ${ }^{2,7}$. Generally, these techniques are based on reversible switching between two states. Whereas STED is based on a deterministic switching in a nanometric interference pattern, STORM and PALM are based on wide-field illumination and stochastic switching on the level of single isolated molecules, which are then localized. Other approaches, such as super-resolution optical fluctuation imaging $(\mathrm{SOFI})^{8}$, reversible saturable optical fluorescence transitions (RESOLFT) microscopy 9,11 and saturated structured illumination microscopy $(\text { SSIM })^{12,13}$, are also based on stochastic or deterministic switching between two states and provide spatial resolution enhancement.
Here we present an alternative approach that distinguishes adjacent molecules or nanoareas in the sample (arranged, for example, in a grid of $50 \mathrm{~nm} \times 50 \mathrm{~nm}$ rectangular areas) by different average orientations of fluorescent dyes attached to rigid sample structures within these nanoareas. This is done by rotating the polarization of a wide-field excitation beam and detecting the periodic signals emitted with different phases from different nanoareas using wide-field camera detection (SPoD). We also show that the range of polarization angles that results in effective excitation of differently oriented molecules can be substantially narrowed by rotating a second wide-field de-exciting stimulated emission beam of a polarization perpendicular to the excitation beam polarization (ExPAN), resulting in better spatial resolution of overlapping fluorescent molecules.

Whereas SOFI or other blinking-based methods must rely on random fluorescence fluctuations occurring at the timescale of the camera frame rate, SPoD controls the fluorescence modulation frequency of standard dyes so that it ideally matches the camera frame rate. In addition, the periodic recurrence of the signals allows the signal-to-noise ratio to be improved by averaging several periods and thereby enhancing the ability to distinguish nanoareas in a manner similar to lock-in detection ${ }^{14,15}$. Unlike techniques based on single-molecule detection, in which it is essential to detect only very isolated photoswitchable single molecules, SPoD also allows bundled signals of several standard fluorescent molecules with different modulation phases to be distinguished in adjacent nanoareas. This avoids large numbers of complete on-off cycles or high intensities and is less dependent on using samples labeled at specific densities. It also avoids using optical setups with very small detection volumes and low background signals, such as total-internal-reflection fluorescence (TIRF) microscopes.

Because of the background-reducing effect of lock-in approaches, the method can be employed at deeper penetration depths than are typically possible with super-resolution techniques. The approach works for all standard fluorescent samples

\footnotetext{
${ }^{1}$ Department of Biophysical Chemistry, Institute for Physical and Theoretical Chemistry, University of Braunschweig, Braunschweig, Germany. ${ }^{2}$ Biomolecular Spectroscopy and Single-Molecule Detection Group, Max Planck Institute for Biophysical Chemistry, Göttingen, Germany. ${ }^{3}$ Felix Bernstein Institute for Mathematical Statistics in Bioscience, Göttingen, Germany. ${ }^{4}$ Institute for Mathematical Stochastics, University of Göttingen, Göttingen, Germany. ${ }^{5}$ Statistical Inverse Problems in Biophysics Group, Max Planck Institute for Biophysical Chemistry, Göttingen, Germany. ${ }^{6}$ Division of Cellular Neurobiology, Zoological Institute, University of Braunschweig, Braunschweig, Germany. ${ }^{7}$ Institute of Organic and Biomolecular Chemistry, University of Göttingen, Göttingen, Germany. ${ }^{8}$ These authors contributed equally to this work. Correspondence should be addressed to P.J.W. (pwalla@gwdg.de).
}

RECEIVED 8 JULY 2013; ACCEPTED 7 MARCH 2014; PUBLISHED ONLINE 6 APRIL 2014; DOI:10.1038/NMETH.2919 
in which fluorescent dyes attached to stable (biological) structures have different average orientations.

\section{RESULTS}

Because linear polarized light preferably excites molecules having a transition dipole moment orientation parallel to its polarization (Fig. 1a), rotating the polarization results in oscillating fluorescence signals generated by the molecules ${ }^{16,17}$ (Fig. 1b). As molecules attached to different places at particular structures have different random average orientations (Fig. 1c), the maximum contribution of each to the fluorescence image occurs at different times or with different phases (Fig. 1d). Thus, even if structural details are hidden in standard fluorescence images owing to diffraction-limited spatial overlapping, recording the periodical signals from the different emitters yields additional information that allows differentiating them and tracing back their original positions. A more distinct periodic signal is obtainable by narrowing the orientation angle range of molecules excited by linear polarized light. This can be achieved by irradiation of the sample with a second wide-field de-exciting stimulated emission beam that also rotates but has a linear polarization that is always exactly perpendicular to the polarization of the exciting light (ExPAN). The de-exciting beam prevents emission from all molecules except those with transition dipole moments oriented perpendicular to the second beam (Fig. 1b); in other words, only those molecules with transition dipole moments exactly parallel to the polarization of the exciting beam can fully emit fluorescence. Consequently, during rotation of both beams, the molecules flash quickly and periodically after each other only at times when the polarization of the excitation exactly matches their transition dipole moment (Fig. 1b,d). The approach is technically simple because it does not require the generation of nanometric structures of light in the focal area and can be applied in the wide field.

High-resolution structure information can also be gained from the modulation signals at higher label densities when individual molecules cannot be distinguished within a diffraction-limited area by different phases (Fig. 1e-h). At higher label densities, bundled signals from several fluorescent moleculesfor example, in a nanoarea of $50 \mathrm{~nm} \times 50 \mathrm{~nm}$ corresponding to the detection pixel size (Fig. 1e,f)-are distinguished by their different periodic sum signals and phases (Fig. 1g). For example, the average orientations of several molecules in separate nanoareas can be deduced from a simple vector addition (Fig. 1e). Without ExPAN, the signals emitted from such nanoareas (Fig. 1g) are similar to the signals of single molecules, which are cosine-squared $\left(\cos ^{2}\right)$ functions of the timedependent rotation angle, $\alpha(t)$ (Fig. 1d). They are also angle- and, thus, time-dependent $\cos ^{2}$ functions that are maximal when the excitation polarization matches the average orientation (Fig. 1e) in that nanoarea. The main difference is that the fluorescence signal modulates around a higher average intensity that is proportional to the number of molecules, $N_{\text {mol }}$, per nanoarea.
Figure 1 | Setup and principle of SPoD and ExPAN. (a) Setup. In SPoD, the excitation polarization in a wide-field fluorescence microscope (turquoise wave) is rotated with a fixed frequency by using, for example, a $\lambda / 2$-wave plate (WP). In ExPAN, an additional wide-field de-excitation beam (red wave) is rotated with a polarization perpendicular to the excitation beam (red arrows). $\alpha$, angle between the excitation light polarization (turquoise vector above microscope objective) and a molecule's transition dipole moment vector (black arrow). (b) Dependence of a molecule's fluorescence intensity, $I$, on $\alpha$ with and without ExPAN shown in a polar grid and in a Cartesian plot. Without ExPAN: dashed dumbbell-shaped turquoise line in polar grid and black function in Cartesian plot. With ExPAN: solid, dumbbellshaped turquoise function in polar grid and red line in the Cartesian plot. Red dumbbell-shaped function in polar grid: de-excitation probability of the ExPAN beam as function of $\alpha$.

(c,d) Different molecules attached to rigid structures (visualized here by three dumbbells 1-3 of labels attached to one of three spherical objects) having different average orientations (c). Their maximum fluorescence intensity contribution, $I(t)$, to the fluorescence image occurs periodically at different times, $t$, or angles, $\alpha(t)(\mathbf{d})$. (e) Molecule dipole moment vectors (orange) and vector addition or average orientation of the dipole moments (turquoise)
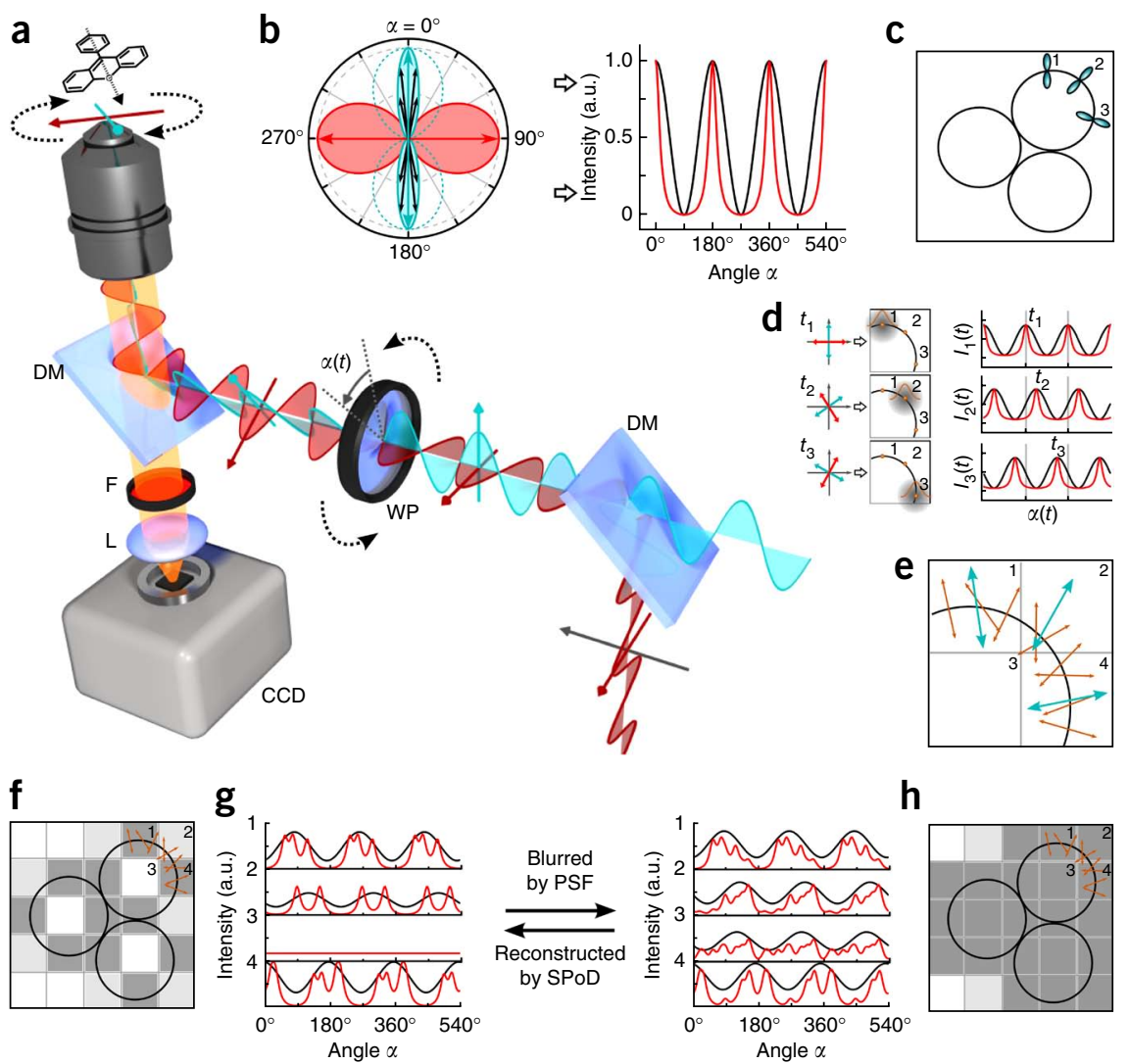

h

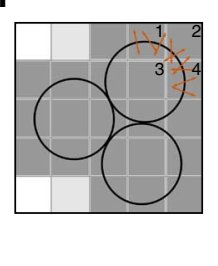

of more than one molecule in detection pixel-sized nanoareas (1-4). (f) Fluorescence intensity (gray squares) emitted from nanoareas containing more than one molecule. (g) Periodic signals emitted from nanoareas 1-4 (black, without ExPAN; red, with ExPAN) and blurred according to the point spread function (PSF) over all detection pixels. (h) Fluorescence intensity (gray squares) emitted from all nanoareas blurred over the detection pixels by the PSF. DM, dichroic mirror; F, fluorescence filter; L, lens; CCD, charge-coupled device; a.u., arbitrary units. 

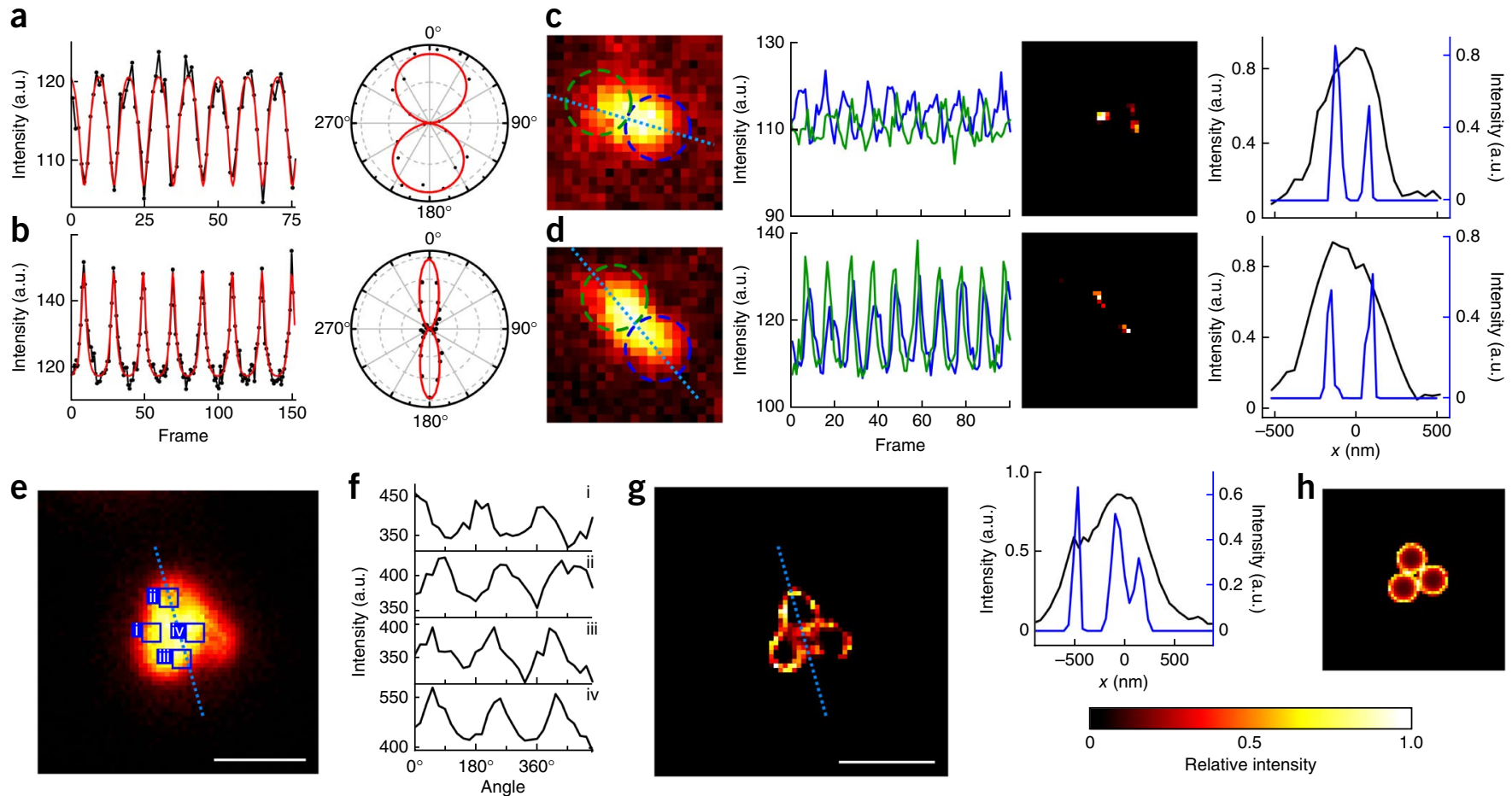

Figure 2 | Experimental SPoD and ExPAN data from single molecules and surface-labeled nanospheres. (a,b) Modulation signals due to 568-nm excitation of a single Atto 590 molecule without (a) and with (b) 715-nm ExPAN beam. Black dots, experimental data; red lines, theoretical function (equation (3), Online Methods) fit to the data. (c,d) Average intensity images, modulation signals, high-resolution images and intensity profile plots of molecule pairs observed without (c) and with (d) ExPAN. Blue and green modulation curves represent time-dependent average signals from the corresponding blue and green dashed circles in the images as observed in a series of camera frames. Black and blue intensity profile plots are from the diffraction-limited and high-resolution image, respectively. (e-g) Diffraction-limited image (e), modulation signals from selected areas (f) and highresolution image and profile plot $(\mathbf{g})$ from three nanospheres (diameter, $500 \mathrm{~nm}$ ) surface labeled with Atto 590 . (h) Simulation of a high-resolution image of surface-labeled nanospheres (projection of many labels at three sphere surfaces onto a single image plane). Scale bars, $1 \mu \mathrm{m}$. a.u., arbitrary units.

In addition, the modulation amplitude is, in first approximation, inversely proportional to $\sqrt{N_{\text {mol }}}$ in such a nanoarea because for increasing molecule numbers, the modulation signals from individual emitters are increasingly averaged. In the raw image these signals are blurred over several pixels by the point spread function (PSF) (Fig. 1g,h), but analysis of the modulation amplitudes, phases and average intensities detected in each pixel can be used to reconstruct the original density of molecules in each nanoarea (Fig. 1f,g). In order to differentiate neighboring nanoareas in the presence of background noise, a sufficiently large modulation signal can be achieved either by averaging the modulation over several periods (similarly to lock-in detection, which is also based on the periodic recurrence of signals in the presence of a large background ${ }^{14,15}$ ) or by using ExPAN.

\section{Validation using single molecules and labeled nanospheres} To demonstrate the ExPAN effect experimentally, we first detected signals from single molecules on a coverslip with and without ExPAN (Fig. 2a,b). Application of ExPAN notably reduced the duration for which the molecules emitted fluorescence. The intensity of the stimulated emission beam was $\sim 1.7 \mathrm{MW} / \mathrm{cm}^{2}$ for all ExPAN experiments shown here ${ }^{18}$. In principle, the duration for which a single molecule fluoresces can be reduced as desired by changing the power of the stimulated emission beam (see Online Methods), but the intensities described above usually sufficed because the demodulation approach does not require a complete on-and-off behavior of the signals from individual emitters.
Next we investigated molecule pairs separated by subdiffraction distances but with differing orientation differences (Fig. 2c,d and Supplementary Fig. 1). When the molecule orientations differed by a $\Delta \alpha$ of $\sim 70^{\circ}$, application of SPoD demodulation image analysis (Supplementary Note 1) clearly showed the positions of both molecules with high resolution even though there was notable spatial overlap between the signals as well as small oscillation amplitudes. When the orientation of both molecules was very similar $\left(\Delta \alpha \approx 20^{\circ}\right)$, ExPAN could still resolve the molecules.

To test the performance of SPoD with ExPAN when imaging molecules at higher densities, we imaged three surface-labeled nanospheres stuck on a coverslip for which the high-resolution structure was known. We could distinguish distinct modulation signals from selected pixel areas that were caused by the different molecule orientations in the selected areas (Fig. 2e,f). Use of SPoD with ExPAN to measure signals from a raster of individual $50 \mathrm{~nm} \times 50 \mathrm{~nm}$ nanoareas corresponding to the detection pixel size allowed us to generate a super-resolution image (Fig. 2g) that shows details, such as the gap between the three spheres, that can be resolved neither in a diffraction-limited fluorescence image nor in its deconvolution image (Supplementary Fig. 2b). The image is highly similar to a simulation of a high-resolution image of surface-labeled nanospheres (Fig. 2h) that was obtained by projecting many labels at a sphere surface onto the image plane. Because out-of-focus background and signals from nonfocused molecules above or below the focal plane overlap notably, their contribution to the modulation amplitude decreases quickly 
a

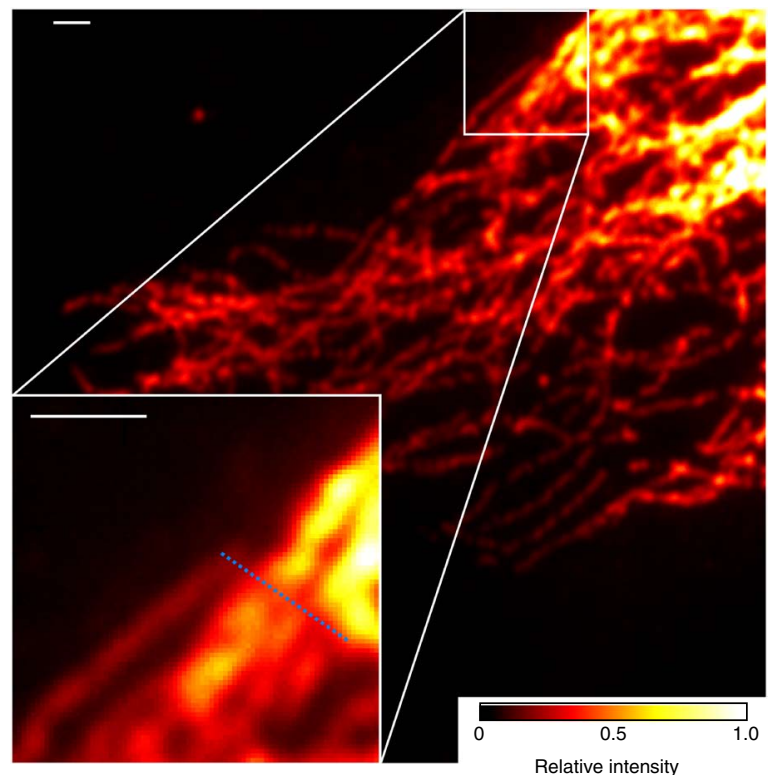

b

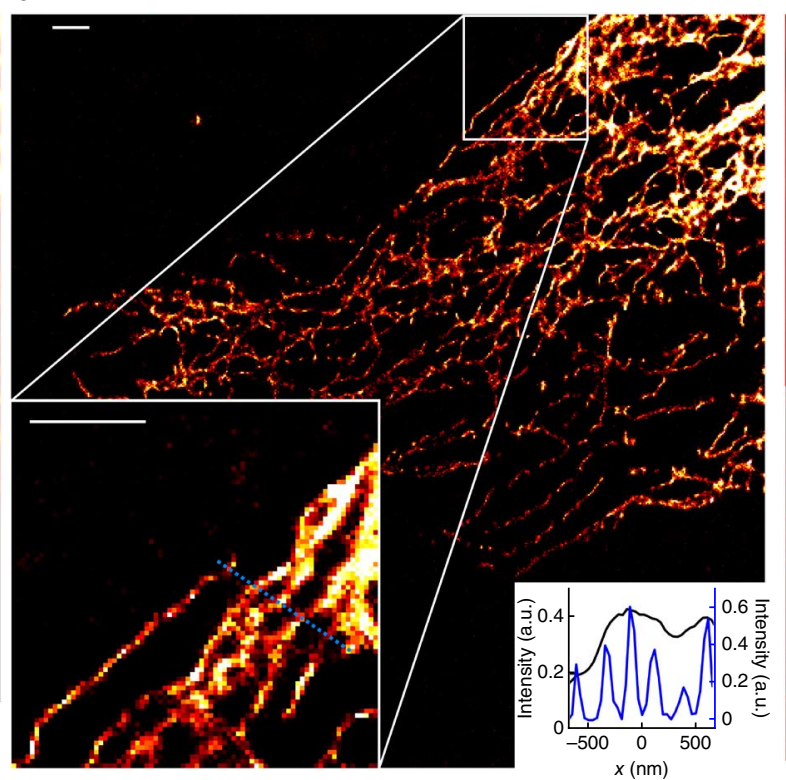

C

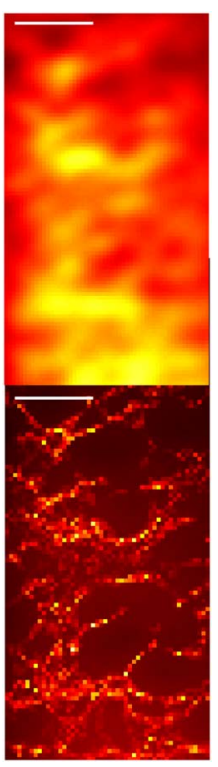

Figure 3 | Images from immunolabeled microtubules. (a,b) Diffraction-limited (a) and regular SPoD image (b) of microtubules on a coverslip (Alexa 488 labeled; $\lambda_{\text {exc }}=488 \mathrm{~nm}$; data acquisition time, $1 \mathrm{~s}$ ). (c) Sample of more dense fibers and out-of-focus background for which ExPAN was additionally used (Atto 590 labeled; top, diffraction-limited image; bottom, SPoD image; excitation wavelength, $\lambda_{\mathrm{exc}}=568 \mathrm{~nm}$; de-exciting ExPAN wavelength, $\lambda_{\mathrm{SE}}=715 \mathrm{~nm}$; data acquisition time, $670 \mathrm{~ms})$. Scale bars, $1 \mu \mathrm{m}$. a.u., arbitrary units.

owing to averaging, resulting in improved background reduction and $z$ sectioning. We also note that there are no dyes at the bottom of the spheres because they were labeled after they were attached to the coverslip. For both these reasons, the experimental data (Fig. 2g) appear darker in the middle of the spheres than the simulated $z$ projection of entire spheres.

Using the width of Gaussian fits to the smallest peaks in profile plots across the bead images as a basis, we estimated that the resolution of the densely labeled spheres achieved using ExPAN ( 40-nm full-width at half-maximum (FWHM)) was about six times better than the resolution of the diffraction-limited image (Fig. 2e-g). We also estimated the resolution achieved by SPoD with or without ExPAN, using a theoretical first-order approximation (Supplementary Note 2). With typical parameters and densities of fluorescent markers, the theoretical expectation is that data acquisition times of less than $1 \mathrm{~s}$ suffice to achieve a resolution on the order of the pixel size $(q=48 \mathrm{~nm})$, which corresponds well to our experimental observations. The PSF of the method has dimensions in the $x, y$ direction corresponding to this resolution and in the $z$ dimension approximately that of the wide-field microscope, albeit reduced similarly to in SOFI $(\sim 1 \mu \mathrm{m})$. If single molecules can be separated by their phase (with or without ExPAN; Fig. 2a-d), the resolution becomes identical to the localization precision achievable by PALM and STORM $(\sim 16 \mathrm{~nm})$.

\section{Images from biological structures}

In biological or other samples, the orientation of fluorescent markers may fluctuate on timescales that are comparable to or faster than the frame rate in video microscopy. However, as already demonstrated by the examples of the fluorescent markers attached to the nanosphere surfaces (Fig. 2e,f) and exposed to an aqueous environment, often the average orientation persists longer when the markers are attached to more rigid structures.
Consequently, it should be possible to modulate probes attached to rigid biological structures such as the cytoskeleton by using our polarization approach ${ }^{19,20}$.

To explore the use of SPoD to image biological samples, we first compared conventional wide-field (Fig. 3a) and SPoD wide-field (Fig. 3b) imaging of Alexa 488-immunolabeled microtubule fibers in fixed PtK2 cells. The SPoD images clearly show details such as closely spaced fibers that are distinguishable neither in the diffraction-limited image nor in an image derived by deconvolution (Supplementary Fig. 2e). We estimated the resolution at $\sim 40-50 \mathrm{~nm}$ on the basis of the FWHM of profile plot peaks. Another sample immunolabeled with Atto 590 displayed much higher fiber density and strong out-of-focus background in the conventional wide-field image (Fig. 3c), but use of SPoD—and, additionally, ExPAN - still allowed us to resolve individual fibers.

For typical dye parameters and concentrations (more than five molecules per nanoarea, as derived from the total fluorescence intensity), equation (11) in Supplementary Note 2 predicts variations in the resolution at different places of the sample smaller than $\sim 10-20 \%$. Indeed, a comparison of our microtubule images with images obtained by other super-resolution techniques ${ }^{2,21}$ demonstrates that this resolution variation is similar to that observed with STED or PALM, which are also subject to local sample variations in fluorescence lifetimes or label densities. Nevertheless, it is always advantageous to choose an image pixel size that is similar to or somewhat larger than the worst local resolution in the entire image, thereby ensuring a homogeneous resolution throughout the image. Closer inspection of the phase information contained in the modulated images suggests that the orientations of the labels reflect a more or less regular, helical structure around the microtubules.

Next we explored the use of SPoD to image membrane-labeled neurons in brain-slice cultures. We used biolistic transfection of 
a
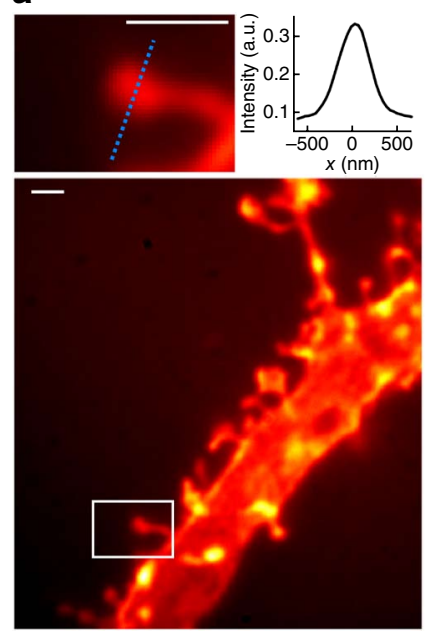
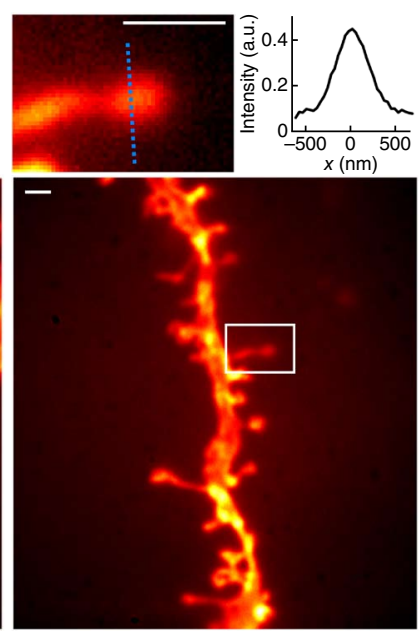

b
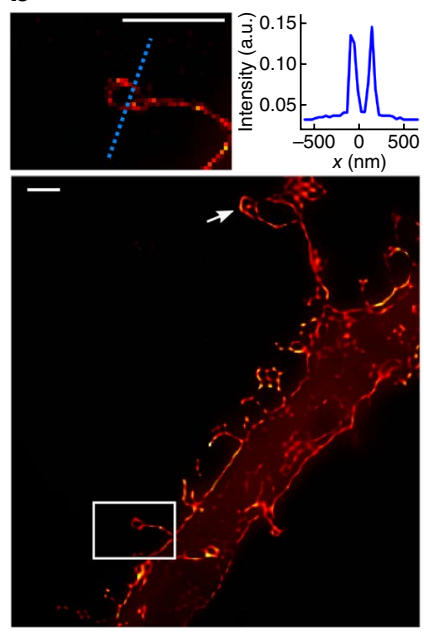
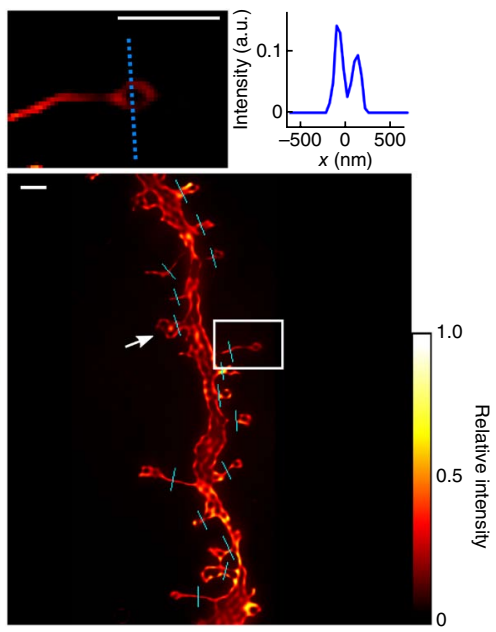

Figure 4 | Images from fEGFP membrane-labeled hippocampal neurons in organotypic slice culture. (a) Diffraction-limited images of fEGFP membranelabeled hippocampal neurons $\left(\lambda_{\text {exc }}=488 \mathrm{~nm}\right.$ ). Boxed areas are shown magnified at the top. (b) Corresponding SPoD images. The data acquisition time was $330 \mathrm{~ms}$ for the left spine with profile plot and $3.3 \mathrm{~s}$ for the right spine with profile plot. The arrows point to spine heads containing a spine apparatus. The dotted blue lines spanning spine necks indicate regions used for measurements of neck width. Scale bars, $1 \mu$ m. a.u., arbitrary units.

organotypic hippocampal slice cultures to label the membrane of dendritic stretches of pyramidal neurons with a farnesylated form of enhanced GFP (fEGFP). We again compared conventional wide-field imaging (Fig. 4a) and wide-field SPoD imaging (Fig. 4b) of the membrane structure of single spine heads (the postsynaptic side of excitatory synapses in the hippocampus). Although individual spines were clearly resolved in both cases, only SPoD could resolve the membrane structure of single spine heads. A data acquisition time of $330 \mathrm{~ms}$ yielded very similar results to an acquisition time of $3.3 \mathrm{~s}$, just being slightly noisier (Fig. 4).

To assess the quality of the image data, we analyzed the diameters of 16 spine necks (Fig. $\mathbf{4 b}$ ). This yielded a median value of $100 \mathrm{~nm}$ and a total range of spine neck diameters from 60 to $370 \mathrm{~nm}$. An analysis of the spine-head diameters of the same spines yielded a median value of $380 \mathrm{~nm}$ and a range of spine-head diameters from 70 (this value is actually the thickest diameter of the smallest dendritic filopodium) to $710 \mathrm{~nm}$. These values are in good agreement with the dimensions known from serialsection electron microscopy and other super-resolution images in the literature $22-26$.

It is known from serial-section electron microscopy images ${ }^{25}$ that larger spine heads can contain membrane-rich organelles such as the spine apparatus in the spine-head center. Intriguingly, we observed similar structures in the center of some spine heads (Fig. 4b) that were not visible through deconvolution of conventional wide-field images (Supplementary Fig. 2h). Sometimes this spine apparatus structure was already apparent in the raw modulation data in the form of phases that were clearly different from phases of the surrounding membrane parts.

The distinctive 'edges' of the membranes in the SPoD spine images might seem surprising in the absence of subdiffraction optical sectioning, but other high-resolution membrane-labeling studies confirm this appearance ${ }^{26}$. This is similar to the edges in the images of the well-defined surface-labeled nanospheres (Fig. 2g) for which the expected high-resolution image appearance was exactly confirmed (Fig. 2h). All membrane parts that appear with diffraction-limited resolution in the standard wide-field image
(Fig. 4a) also appear with high $x$ and $y$ resolution in the SPoD images as maximum-intensity projections of the $z$ range of the microscope (including all spines that fall into that range). Because the label density is highest along the edges in such a projection, they appear most prominently in both diffraction-limited and high-resolution images, but this effect is especially noticeable in high-resolution images.

Finally, we briefly tested whether the SPoD method could be combined with two-photon laser scanning microscopy to improve the penetration depth. We used a special objective for deep-tissue two-photon microscopy of fEGFP-labeled neurons in fixed brain slices and observed spine heads at $\sim 0.4-$ and $\sim 0.6-\mathrm{mm}$ depths (Supplementary Fig. 3).

\section{DISCUSSION}

We have demonstrated that SPoD allows the observation of highresolution images of standard samples on short timescales. Special sample preparations are not mandatory, and different standard labels and excitation sources work equally well. Wide-field imaging is possible without the need to generate interference structures, and the background-reducing effect of the demodulation techniques permit deeper penetration depths than do TIRF-based approaches. The use of two-photon excitation allows even greater penetration depths. The method requires dyes to be attached to stable structures possessing different preferred average orientations, but this condition was easily satisfied as experimentally confirmed by data from labeled cytoskeleton structures, labeled membranes and labeled surfaces of nanospheres or coverslips. This can be rationalized by the extremely small probability that all fluorophores have the same orientation in diffraction-limited zones of labeled samples. The approach is limited by the ability to detect fluorescence modulation, which might be weak for high fluorophore densities or highly dynamic structures. However, in case of high fluorophore densities, ExPAN can help increase the modulation contrast or, similarly to in lock-in detection, the modulation can be continued until it can be clearly detected above the noise. A notable advantage of the approach over pure stochastic 
methods is that the modulation frequency can exactly be controlled so that it optimally fits to the camera frame rate. Even though heterogeneities are to be expected in the ability to distinguish different label orientations (see, for example, the image simulation in Supplementary Fig. 1), the experimental examples presented here (Figs. 2-4) show that the observed high-resolution images are at least as homogeneous as those of other high-resolution techniques ${ }^{2,21,26}$. In the future, the approach could be extended to three-dimensional demodulation including the $z$ axis of the molecular orientation. This would further increase the spatial and/or time resolution and reduce the PSF in the $z$ direction. The method could also be extended to the analysis of orientational fluctuations as an additional parameter for resolution improvement similarly to the way SOFI uses single-molecule fluorescence fluctuations for resolution enhancement. Finally, ExPAN could also be used to accurately determine the orientation of fluorescent markers attached to structures and measure changes caused by biological or other processes.

\section{METHODS}

Methods and any associated references are available in the online version of the paper.

Note: Any Supplementary Information and Source Data files are available in the online version of the paper.

\section{ACKNOWLEDGMENTS}

This work was financially supported by the Deutsche Forschungsgemeinschaft (DFG) (INST 188/334-1 FUGG). 0.M.S. thanks the International Max Planck Research School (IMPRS) "Physics of Biological and Complex Systems" for financial support. T.A., A.M., C.S. and P.J.W. acknowledge support by DFG Collaborative Research Center (CRC) 755 and 803. M.Z. and M.K. acknowledge support by DFG grant K0 1674/5-1. A.M. acknowledges support by DFG and Schweizerischer Nationalfonds (SNF) grant FOR 916.

\section{AUTHOR CONTRIBUTIONS}

N.H., M.G. and L.S.v.d.H. designed and performed experiments, analyzed data and wrote the paper; T.A. developed the statistical model and analytical tools, wrote the software package SPEED, analyzed data and wrote the paper; J.-H.C. performed experiments; M.Z., 0.M.S., C.S. and M.K. provided samples and edited the manuscript.; A.M. developed the statistical model and analytical tools and edited the paper; and P.J.W. designed experiments, analyzed data and wrote the paper.

\section{COMPETING FINANCIAL INTERESTS}

The authors declare competing financial interests: details are available in the online version of the paper.

Reprints and permissions information is available online at http://www.nature. com/reprints/index.html.

1. Hell, S.W. Microscopy and its focal switch. Nat. Methods 6, 24-32 (2009).

2. Bates, M., Huang, B. \& Zhuang, X. Super-resolution microscopy by nanoscale localization of photo-switchable fluorescent probes. Curr. Opin. Chem. Biol. 12, 505-514 (2008).

3. Lippincott-Schwartz, J. \& Manley, S. Putting super-resolution fluorescence microscopy to work. Nat. Methods 6, 21-23 (2009).
4. Hell, S.W. \& Wichmann, J. Breaking the diffraction resolution limit by stimulated emission: stimulated-emission-depletion fluorescence microscopy. Opt. Lett. 19, 780-782 (1994).

5. Betzig, E. et al. Imaging intracellular fluorescent proteins at nanometer resolution. Science 313, 1642-1645 (2006).

6. Hess, S.T., Girirajan, T.P.K. \& Mason, M.D. Ultra-high resolution imaging by fluorescence photoactivation localization microscopy. Biophys. J. 91, 4258-4272 (2006).

7. Rust, M.J., Bates, M. \& Zhuang, X. Sub-diffraction-limit imaging by stochastic optical reconstruction microscopy (STORM). Nat. Methods 3, 793-796 (2006).

8. Dertinger, T., Colyer, R., Iyer, G., Weiss, S. \& Enderlein, J. Fast, background-free, 3D super-resolution optical fluctuation imaging (SOFI). Proc. Natl. Acad. Sci. USA 106, 22287-22292 (2009).

9. Chmyrov, A. et al. Nanoscopy with more than 100,000 'doughnuts'. Nat. Methods 10, 737-740 (2013).

10. Geisler, C. et al. Drift estimation for single marker switching based imaging schemes. Opt. Express 20, 7274-7289 (2012).

11. Hell, S.W. Toward fluorescence nanoscopy. Nat. Biotechnol. 21, 1347-1355 (2003).

12. Heintzmann, R., Jovin, T.M. \& Cremer, C. Saturated patterned excitation microscopy - a concept for optical resolution improvement. J. Opt. Soc. Am. A Opt. Image Sci. Vis. 19, 1599-1609 (2002).

13. Gustafsson, M.G.L. Nonlinear structured-illumination microscopy: wide-field fluorescence imaging with theoretically unlimited resolution. Proc. Natl. Acad. Sci. USA 102, 13081-13086 (2005).

14. Marriott, G. et al. Optical lock-in detection imaging microscopy for contrast-enhanced imaging in living cells. Proc. Natl. Acad. Sci. USA 105, 17789-17794 (2008).

15. Richards, C.I., Hsiang, J.-C. \& Dickson, R.M. Synchronously amplified fluorescence image recovery (SAFIRe). J. Phys. Chem. B 114, 660-665 (2010).

16. Lakowicz, J.R. Principles of Fluorescence Spectroscopy 2nd edn. (Kluwer/Plenum, 1999).

17. Walla, P.J. Modern Biophysical Chemistry: Detection and Analysis of Biomolecules (Wiley, 2009).

18. Vicidomini, G. et al. Sharper low-power STED nanoscopy by time gating. Nat. Methods 8, 571-573 (2011).

19. Mirzov, 0. et al. Polarization portraits of single multichromophoric systems: visualizing conformation and energy transfer. Small $\mathbf{5}$, 1877-1888 (2009)

20. Thomsson, D., Lin, H. \& Scheblykin, I.G. Correlation analysis of fluorescence intensity and fluorescence anisotropy fluctuations in singlemolecule spectroscopy of conjugated polymers. ChemPhysChem 11, 897-904 (2010).

21. Hein, B., Willig, K.I. \& Hell, S.W. Stimulated emission depletion (STED) nanoscopy of a fluorescent protein-labeled organelle inside a living cell. Proc. Natl. Acad. Sci. USA 105, 14271-14276 (2008).

22. Harris, K.M. \& Kater, S.B. Dendritic spines: cellular specializations imparting both stability and flexibility to synaptic function. Annu. Rev. Neurosci. 17, 341-371 (1994).

23. Urban, N.T., Willig, K.I., Hell, S.W. \& Nägerl, U.V. STED nanoscopy of actin dynamics in synapses deep inside living brain slices. Biophys. J. 101, 1277-1284 (2011).

24. Harris, K.M., Jensen, F.E. \& Tsao, B. Three-dimensional structure of dendritic spines and synapses in rat hippocampus (CA1) at postnatal day 15 and adult ages: implications for the maturation of synaptic physiology and long-term potentiation. J. Neurosci. 12, 2685-2705 (1992).

25. Deller, T. et al. Synaptopodin-deficient mice lack a spine apparatus and show deficits in synaptic plasticity. Proc. Natl. Acad. Sci. USA 100, 10494-10499 (2003).

26. Izeddin, I. et al. Super-resolution dynamic imaging of dendritic spines using a low-affinity photoconvertible actin probe. PLOS ONE 6, e15611 (2011). 


\section{ONLINE METHODS}

Test of demodulation with simulated data of single-molecule pairs and membrane-like structures. Before we demodulated the signals of biological samples and single molecules, we tested the demodulation algorithm (see Supplementary Note 1) using simulated data. In a first test we investigated the results obtained with this algorithm using simulations of single molecules that have different distances and different relative orientations and thus phases (Supplementary Fig. 1). The simulated data considered background noise, photon shot noise and statistical dark-state blinking of the molecules and are based on parameters similar to those of the experimental data shown in Figure 2 (see "Generation of simulated data" below). The algorithm can localize the position of the molecules at distances that are far smaller than would be possible on the basis of diffraction-limited images or deconvolution (Supplementary Fig. 1). Only for small phase differences $\left(\Delta \alpha \leq 30^{\circ}\right)$ and the smallest distances is the algorithm capable of merely deconvoluting the image instead of full resolution.

We also simulated modulation data from statistically positioned and oriented dyes in sectional views of membranes of more complicated shapes (Supplementary Fig. 4). Analyzing these data in the same way as the experimental data shown in Figures 2-4 was suitable for determining the original structure of the labeled membrane (Supplementary Fig. 4b). Even a single period (10 frames of $33 \mathrm{~ms}$ ) was sufficient to obtain this result. Also, analyzing a simulation of notably higher dye concentrations, resulting in much smaller modulation, was still suitable for determining the original structure (Supplementary Fig. 4c).

Mathematical model describing modulated signals using ExPAN. The fluorescence emission of a two-state system with a ground state $S_{0}$ and an excited state $S_{1}$ and taking stimulated emission into account can be described by the rate constant for excitation $k_{\text {exc }}$, the fluorescence decay rate $k_{\mathrm{fl}}$ and the rate constant for stimulated emission $k_{\mathrm{SE}}$. Assuming equilibrium conditions, the population of the excited state, $\mathrm{S}_{1}$, is $N_{1}=k_{\text {exc }}\left(k_{\text {exc }}+k_{\mathrm{fl}}+k_{\mathrm{SE}}\right)$. If excitation and stimulated emission beams are linearly polarized, $k_{\text {exc }}$ and $k_{\mathrm{SE}}$ are functions of the angle $\alpha$ between the transition dipole moment and polarization orientation:

$$
\begin{aligned}
& k_{\mathrm{exc}}(\alpha)=k_{0, \mathrm{exc}} \cos ^{2}(\alpha) \\
& k_{\mathrm{SE}}(\alpha)=k_{0, \mathrm{SE}} \cos ^{2}(\alpha)
\end{aligned}
$$

In the case of ExPAN, the beams have perpendicular polarizations and for $N_{1}$ results:

$$
N_{1}(\alpha)=\frac{k_{0, \mathrm{exc}} \cos ^{2}(\alpha)}{k_{\mathrm{fl}}+k_{0, \mathrm{exc}} \cos ^{2}(\alpha)+k_{0, \mathrm{SE}} \cos ^{2}\left(\alpha+\frac{\pi}{2}\right)}
$$

Because the population of the $S_{1}$ state is proportional to the fluorescence output, this equation describes the shape of the resulting ExPAN signal as shown in Figures $\mathbf{1 b}, \mathbf{d}$ and $\mathbf{2 b}, \mathbf{d}$.

Deconvolution. Deconvolution algorithms as used for the comparison shown in Supplementary Figure 2 were described previously. One of the most prominent ways to deconvolve an image is by maximum-likelihood methods, i.e., by computing the density $\hat{g}(r)$ of molecules at location $r$ that has the highest probability of having generated the observed images. This can be achieved by the Richardson-Lucy (also known as expectation maximization, EM) algorithm ${ }^{27-29}$ or related algorithms $s^{30}$. It is well known, however, that this works well only when the number of parameters to be estimated is much smaller than the number of observations (image pixels) or when the algorithm is stopped at an early iteration stage ${ }^{31,32}$.

Generation of simulated data. The simulated data were generated with Matlab using a point spread function with Gaussian intensity profile and a FWHM of 5 pixels. The simulated image was in total $512 \times 512$ pixels. The polarization-dependent intensity modulation was modeled by $I(\alpha) \propto \cos ^{2}(\alpha)$ or in the case of ExPAN as described by equation $(3)\left(I(\alpha) \propto N_{1}\right)$ with a sampling rate of ten frames per modulation period. Poisson noise was applied to the signal on every pixel, and normally distributed background noise was added. For simulation of dark states, there was a $1 \%$ per frame probability for every molecule to switch between the emitting and non-emitting state. In Supplementary Figure 4, molecule positions and dipole-moment orientations were randomly generated, although molecule positions were restricted to the shown mask. For Supplementary Figure 4a,b 800 molecules were simulated, whereas the high-density simulation in Supplementary Figure 4c was based on a simulation using 8,000 molecules.

Sample preparation. For single-molecule samples, coverslips were thoroughly washed with isopropyl alcohol and distilled water and dried. A droplet of $30 \mu \mathrm{L}$ Atto 590-free acid (Atto-Tec) solution (50 nM in $\mathrm{MeOH}$ ) was placed on the coverslip. After evaporation of the solvent, coverslips were ready for measurement. Before each measurement, a drop of water was added on the top of the coverslip to reduce reflection of the excitation light at the coverslip-air surface. For surface-labeled nanosphere samples, streptavidin-coated nanospheres with a mean diameter of $0.51 \mu \mathrm{m}$ (Bangs Laboratories) were diluted in phosphate-buffered saline (PBS) and immobilized on a bovine serum albumin (BSA)-coated coverslip with $25 \%$ biotin-labeled BSA (Sigma-Aldrich). Excess nanospheres were removed by repeated washing with PBS buffer. A solution of $30 \mathrm{~nm}$ Atto 590-biotin (Atto-Tec) in PBS was added. After a sufficient amount of Atto 590-biotin bound to the microspheres, excess dye was removed by washing with PBS. During the preparation and measurement of the nanosphere samples, the nanospheres as well as labels were kept the entire time in an aqueous environment. The microtubule networks from whole fixed PtK2 cells were immunolabeled with Alexa 488 similarly as in ref. 33. Briefly, mammalian PtK2 cells (male rat-kangaroo kidney epithelial cells, American Type Culture Collection; no authentication was done) were grown on coverslips and fixed for $5 \mathrm{~min}$ with $4 \%(\mathrm{w} / \mathrm{v})$ formaldehyde in PBS, permeabilized with $0.5 \%$ (v/v) Triton X-100 in PBS and blocked with 5\% (w/v) BSA in PBS. $\alpha$-tubules were immunolabeled with anti- $\alpha$-tubulin antibody from mouse (Abcam, ab7750, dilution 1:100). For samples in Figure 3a,b the primary antibody was labeled with Alexa Fluor 488 goat antimouse (Invitrogen, A-10667, dilution 1:200). Samples in Figure 3c were labeled with monoclonal anti-mouse antibodies from rabbit (Abcam, ab125913, dilution 1:100) that were custom conjugated to Atto 590 (Atto-Tec). After labeling, coverslips were mounted using polyvinyl alcohol as mounting medium with DABCO (1,4-diazabi cyclo[2.2.2] octane) added as an antifading agent (Sigma-Aldrich). Hippocampal organotypic cultures were prepared from postnatal 
day 5 (P5) C57BL/6 wild-type (WT) mice (sex undetermined) following the method of Stoppini et al. ${ }^{34}$. Briefly, the hippocampus was dissected in ice-cold sterile Gey's balanced salt solution and sliced transversely at a thickness of $400 \mu \mathrm{m}$. The slices were plated onto Millicell CM membrane inserts (Millipore) and cultivated in a $37^{\circ} \mathrm{C}, 5 \% \mathrm{CO}_{2}, 99 \%$ humidity incubator in a DMEM medium containing glucose, donor equine serum and L-glutamine. To reduce the number of non-neuronal cells, we treated the slices for $24 \mathrm{~h}$ with a mixture of antimitotic drugs. After $14 \mathrm{~d}$, in vitro hippocampal slice cultures were transfected with an expression plasmid for a membrane-targeted fEGFP (farnesylated EGFP) by particlemediated gene transfer device Helios Gene Gun System (Bio-Rad). Two days after transfection, the slices were fixed overnight with 4\% PFA and mounted with an antifading water-based mounting medium. For the deep-tissue two-photon experiments, transgenic mice expressing membrane-targeted fEGFP in small subsets of neurons (Thy1-mGFP) were transcardially perfused with $40 \mathrm{~mL}$ of $4 \%$ PFA in $0.12 \mathrm{M} \mathrm{PB}$ (phosphate buffer), $\mathrm{pH}$ 7.2-7.4. The brains were dissected immediately, cut into 500 - to $1,000-\mu \mathrm{m}-$ thick coronal sections with a vibratome (Leica) and stored in the same fixative at $4{ }^{\circ} \mathrm{C}$ and imaged within a few hours. All experiments were conducted in accordance with the applicable European and National regulations (Tierschutzgesetz).

One-photon excitation (OPE) setup with $488 \mathrm{~nm}$. Two different wide-field microscope setups were used to perform the measurements. In setup 1, a 488-nm continuous-wave (CW) laser (sapphire 488-50, Coherent) is used for excitation, whose linear polarized beam is expanded through a telescope system. The beam is passing a rotating $\lambda / 2$-wave plate (achromatic $\lambda / 2$ plate, $400-800 \mathrm{~nm}$, Thorlabs) in order to constantly rotate the polarization plane of the linear polarized light. The rotation is achieved through a chopper wheel (Optical Chopper System, Thorlabs) that is synchronized to the electron-multiplying charge-coupled device (EMCCD) camera (see below). Thereafter, the beam is focused onto the back aperture of the microscope objective (NA $=1.35$ oil immersion, UPlanSApo, $60 \times$, Olympus), which is mounted in an inverted microscope body (IX 71, Olympus). Emission light is passed through a dichroic mirror (beam splitter z $488 \mathrm{RDC}, \mathrm{AHF}$ ) and an emission filter (ET band pass 525/50, AHF). An additional lenses system is used to further magnify the image and focus it on an EMCCD camera (iXonEM+897 back illuminated, Andor Technology).

SPoD with ExPAN setup. ExPAN measurements were carried out on a second setup with a similar configuration but using a 568-nm CW laser (Sapphire 568, Coherent) for excitation. The excitation light is here combined with a $715-\mathrm{nm}$ beam from a Ti:sapphire laser in CW mode (Mira 900, Coherent) through a dichroic mirror. The stimulated emission beam and excitation beam have perpendicular polarizations. In comparison to setup 1 , two $\lambda / 4$-wave plates (Achromatic $\lambda / 4$ plate, $400-700 \mathrm{~nm}$, Newport) are used to rotate polarization instead of one $\lambda / 2$, and a different objective $(\mathrm{NA}=1.3$, Fluar, 100×, Zeiss) was used. Furthermore, a motorized stage (Scan IM, Märzhäuser) in the $x, y$ direction and a piezo stage (Custom Nano-Z200, Mad City Labs) in the $z$ direction was used to position the samples. During acquisition of a video frame, overlapping spots of $\sim 10-\mu \mathrm{m}$ diameter of the excitation and de-excitation light were scanned several times over the entire field of view using a $16 \mathrm{kHz}$ resonant scanner (Electro-Optical Products Corp.).
Deep-imaging with two-photon excitation (TPE). For TPE experiments of deep-tissue imaging, linear polarized light from a pulsed laser $(918 \mathrm{~nm}, 80 \mathrm{MHz}$, Chameleon, Coherent) was used. After beam expansion by a telescope system, the linear polarized beam passes a rotating $\lambda / 2$ plate (achromatic $\lambda / 2$ plate, 680 $1,200 \mathrm{~nm}$, Thorlabs) whose rotation is achieved the same way as described for the OPE path of the 488-nm setup. The collimated linear polarized light continues through a fourth telescope system and is coupled into the microscope using a dichroic mirror and a piezo mirror (S-334.1SL, Physik Instrumente). The excitation light fully illuminates the back aperture of the deep-imaging objective (NA $=1.05$ water immersion, XLPLN 25XWMP, 25×, Olympus), and the emission light passes the dichroic mirror (multiphoton emitter HC 770/SP, AHF) and the emission filter (ET band pass 525/50, AHF). An additional filter is used to purify the emission light from reflected excitation light before being focused onto the EMCCD camera. For image acquisition with TPE, the piezo mirror is used to scan the sample. During rotation of the polarization, the camera was operated with a frame frequency of $30 \mathrm{~Hz}$ and triggered the start of the piezo mirror movement that covered a rectangular area of the sample for each camera frame. Because the piezo can be operated at frequencies up to $300 \mathrm{~Hz}$, this allowed us to cover a rectangular range of $3 \times 3$ diffraction-limited excitation spots.

Software. Image data were recorded using either Solis image capture software (Andor) or $\mu$ Manager microscopy software ${ }^{35}$. Algorithms for image processing were implemented in Python 2.7. The algorithm, exemplary raw data and necessary parameters are available in a single ZIP file (Supplementary Software). All microscopy images were visualized using the "Red Hot" lookup table from ImageJ.

Imaging replicates. Images in the figures were representative of results as follows: Figure 2c: $>10$ replicates (images of different single molecule pairs); Figure 2d, $>3$ replicates (images of different single-molecule pairs); Figure 2e,g, $>3$ replicates (images of different groups of nanospheres); Figure $\mathbf{3 a}, \mathbf{b},>3$ replicates (images of different microtubule networks); Figure 3c, 2 or 3 replicates (images of different microtubule networks); Figure 4: $>3$ replicates (images of different dendritic stretches of neurons).

27. Richardson, W.H. Bayesian-based iterative method of image restoration. J. Opt. Soc. Am. 62, 55-59 (1972).

28. Lucy, L.B. An iterative technique for the rectification of observed distributions. Astron. J. 79, 745-749 (1974).

29. Vardi, Y., Shepp, L.A. \& Kaufman, L. A statistical model for positron emission tomography. J. Am. Stat. Assoc. 80, 8-20 (1985).

30. Bertero, M. \& Boccacci, P. Introduction to Inverse Problems in Imaging (Taylor \& Francis, 1998).

31. Bissantz, N., Mair, B.A. \& Munk, A. A statistical stopping rule for MLEM reconstructions in PET. in IEEE Nucl. Sci. Symp. Conf. Rec. 4198-4200 (IEEE, 2008).

32. Munk, A. \& Pricop, M. in Statistical Modelling and Regression Structures (eds. Kneib, T. \& Tutz, G.) 431-448 (Physica, 2010).

33. Testa, I. et al. Multicolor fluorescence nanoscopy in fixed and living cells by exciting conventional fluorophores with a single wavelength. Biophys. J. 99, 2686-2694 (2010).

34. Stoppini, L., Buchs, P.-A. \& Muller, D. A simple method for organotypic cultures of nervous tissue. J. Neurosci. Methods 37, 173-182 (1991).

35. Edelstein, A., Amodaj, N., Hoover, K., Vale, R. \& Stuurman, N. Curr. Protoc. Mol. Biol. 92, 14.20 (2010). 